A
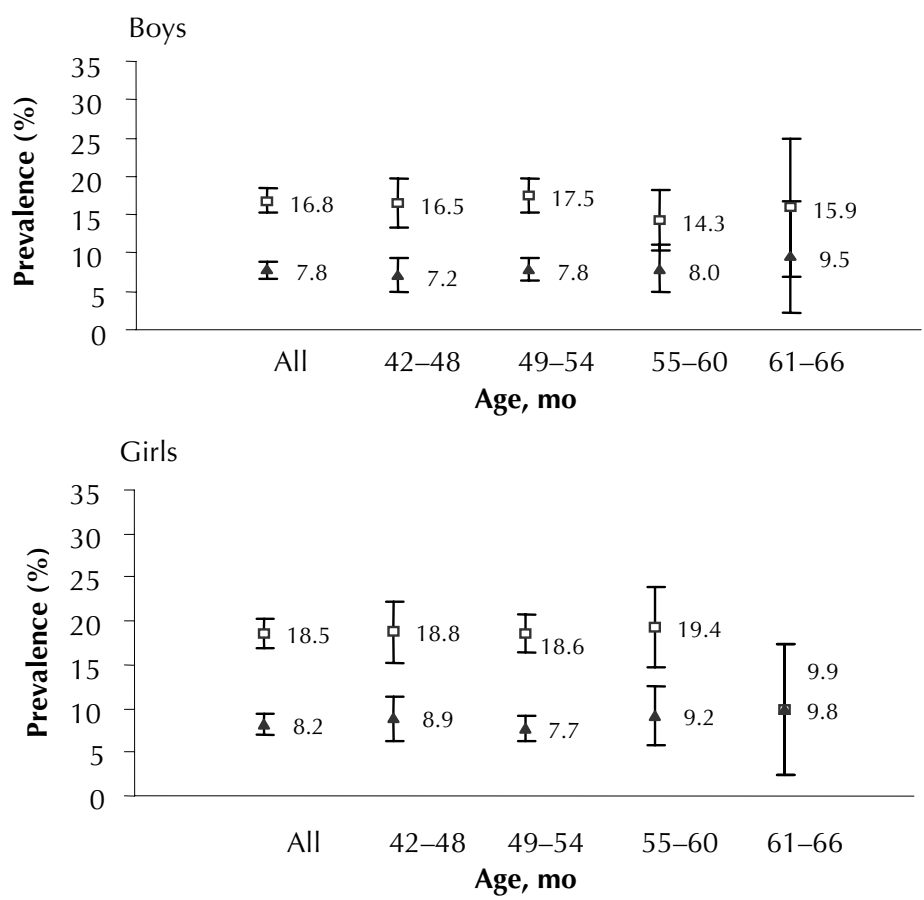

B
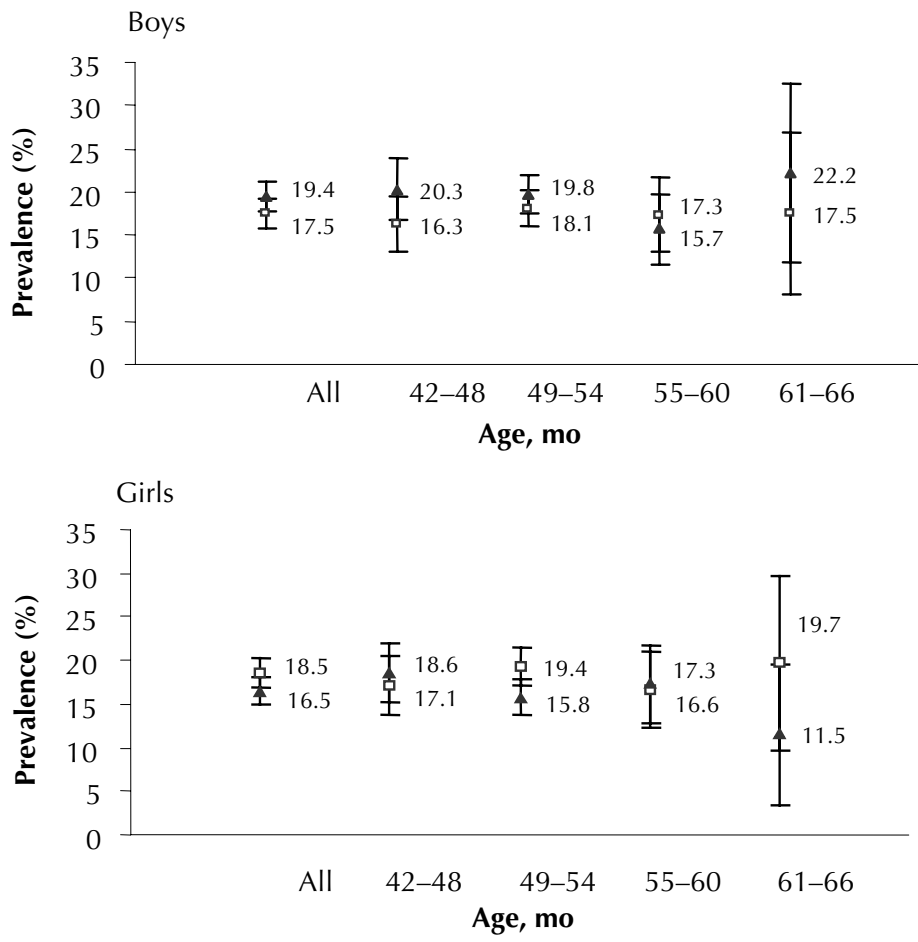

Fig. 1: Prevalence of overweight (squares) and obesity (triangles) among preschool children in Newfoundland and Labrador, by sex and age group, estimated by the methods of Cole and colleagues ${ }^{2}$ (the International Obesity Task Force) (A) and the Centers for Disease Control and Prevention ${ }^{3}$ (B). Error bars represent $95 \%$ confidence intervals.
2. Cole TJ, Bellizzi MC, Flegal KM, Dietz WH Establishing a standard definition for child overweight and obesity worldwide: international survey. BM7 2000;320:1240-3

3. Centers for Disease Control and Prevention. CDC growth charts. Atlanta: Department of Health and Human Services, National Center for Health Statistics; 2000. Report no. 314.

DOI:10.1503/cmaj.1041668

\section{Stats Can under-represents rural shortage}

T suspect that Statistics Canada's inter1 pretation of the data presented in a recent CMAJ news article ${ }^{1}$ underrepresents the shortage of rural practitioners. Marc Hamel of Stats Can feels that the data do not indicate that people in rural areas have more trouble finding a doctor. However, I think the data are misleading, in that practitioners in small communities cannot "close" their practices to new patients - there is simply nowhere else for patients to go. Thus, even though people in rural communities may report that they have a family doctor, there is no mention of how easy it is to get an appointment with that doctor. That rural doctor probably has a significantly longer patient list than the average nonrural practitioner, as well as other responsibilities that decrease the time available for office appointments.

A quick straw poll in my own region of northeastern British Columbia seems to support this. In the 2 larger communities of Fort St. John and Dawson Creek, with several medical clinics serving over 30000 people each, very few doctors are taking new patients. In contrast, in the small communities of Chetwynd, Fort Nelson and Tumbler Ridge (each of which has only one clinic), all doctors are seeing new patients.

Therefore, although at a superficial level it may appear easier for patients to find a family doctor in smaller communities, these communities have significant physician understaffing relative to their larger counterparts and therefore inferior access to care. I don't think it is too much of a stretch to suggest that the apparent small difference in finding a GP between rural and urban settings 
actually under-represents the true difference in access to medical care.

\section{Paul Mackey \\ Rural Physician \\ Fort St. John, BC}

Reference

1. Gagnon L. Stats Can: $14 \%$ of Canadians have no family doctor. CMAf 2004;171(2):124.

Competing interests: Member, Rural Issues Committee of the British Columbia Medical Association.

DOI:10.1503/cmaj.1041293

\section{[Mr. Hamel responds:]}

Tn the 2003 Canadian Community 1 Health Survey, released in June 2004 and summarized in $C M A 7,{ }^{1}$ about $4.5 \%$ of Canadians residing in urban areas and $5.5 \%$ of those in rural areas reported not being able to find a regular medical doctor. Proportionally speaking, these numbers are very similar. However, in terms of population, the $4.5 \%$ in urban areas represented about 965000 of the 1.2 million Canadians (12 years of age or older) who reported that they were unable to find a regular medical doctor.

In analyzing these results, definitions matter. Our analysis was based on the definition of rural areas used for the Canadian census. Thus, we did not differentiate between rural and remote areas, for which the picture might be different. Also, having a regular family doctor does not imply better access to care, and our analysis did not examine the relation between having a regular medical doctor and the process of accessing care. Although we looked at the profile of people with and without a regular medical doctor in terms of some health care services such as routine tests (e.g., blood pressure check, mammography) and use of emergency departments, the survey did not measure issues related to primary care access such as those described by Paul Mackey.

Marc Hamel

Chief, Canadian Community Health Survey

Health Statistics Division

Statistics Canada

Ottawa, Ont.

\section{Reference}

1. Gagnon L. Stats Can: 14\% of Canadians have no family doctor. CMAF 2004;171(2):124.

DOI:10.1503/cmaj.1041703

\section{Not a middle-of-the-road position}

T cannot understand how Dr. Ursus ${ }^{1}$ Lan claim to have a "middle-of-theroad" position on abortion when he clearly supports abortion on demand. He may deeply regret the necessity of abortions; however, by performing these procedures or referring patients for them, he's chosen against his smaller, defenceless patients. $\mathrm{He}$ is on that side of the road.

\section{Donald S. Stephens \\ Physician \\ Stratford, PEI}

\section{Reference}

1. Query. CMAf 2004;171(11):1420.

DOI:10.1503/cmaj.1041752

\section{Strains and toxins of Clostridium}

Tacques Pépin and associates ${ }^{1}$ have re$\int$ ported an epidemic of Clostridium difficile-associated diarrhea (CDAD) associated with an increased case-fatality rate. They hypothesize the presence of a more virulent strain.

The genus Clostridium consists of gram-positive, anaerobic, spore-forming rods and is notorious for causing human and animal diseases by producing various extracellular toxins. $C$. diff $i$ cile exerts its effects through toxin $\mathrm{A}$, an enterotoxin, and toxin B, a cytotoxin, which result in colitis and pseudomembranes. ${ }^{2}$ The development of a more virulent circulating strain could occur, in part, through the acquisition of a novel gut-specific toxin, possibly from another clostridial species.

The manifestations of severe CDAD described by Pépin and associates ${ }^{1}$ (i.e., megacolon, perforation, shock or rapid death) resemble those of another clostridial-related disease, enteritis 